Bull. Chem. Soc. Ethiop. 2018, 32(2), 361-370.

ISSN 1011-3924

(c) 2018 Chemical Society of Ethiopia and The Authors

Printed in Ethiopia

DOI: https://dx.doi.org/10.4314/bcse.v32i2.14

\title{
PREPARATION AND ASSESSMENT OF PROSTAGLANDIN E2 FORMULATED WITH HYDROXYPROPYL METHYL CELLULOSE AND SPAN 60 SUSTAINED RELEASE PESSARIES (VAGINAL SUPPOSITORIES)
}

\author{
Habib Saedi* \\ Polymer Research Unit, College of Science, Al Mustansiriya University, Baghdad, Iraq
}

(Received March 12, 2017; Revised March 24, 2018; Accepted May 2, 2018)

\begin{abstract}
Prostaglandin E2 (PGE2) is presented as a composed release pessaries utilizing two-polymer base. Hydroxypropyl methyl cellulose HPMC was used as a viscosity modifier and bioadhesive agent. Furthermore, nonionic surfactant span 60 (Sp 60) with or without cholesterol $(\mathrm{CH})$ mixed with the base was used. The defined pessaries were assessed with regards to hardness, weight variation, softening time test and in vitro release studies. Every one of the formulations demonstrated consistency with pharmacopoeial benchmarks. In vitro expressed PGE2 disintegration was completed in phosphate buffer $\mathrm{pH} 4.5$ utilizing USP XXII basket apparatus. TEM demonstrated that the PEG/Sp 60 bases formed niosomal vesicles upon hydration with water while the WH15/Sp 60 gave rise to emulsions when melted in phosphate buffer $\mathrm{pH} 4.5$. Both types of the novel composed release pessaries (WH15/Sp 60 and WH15/HPMC) indicated moderate release rates for PGE2 when no CH detected and the recipes WH15/30\%HPMC and PEGIII/SP60 being used as an effective matrix previously to give PGE2 sorting out the release. The outcomes also demonstrated that the $\mathrm{CH}$ addition to either WH15 or PEG base brought about increased PGE2 release rates. No interaction amongst PGE2 and excipients were observed from DSC studies.
\end{abstract}

KEY WORDS: Pessary, PGE2, WH15, HMPC, Sp 60, Polymer, Sustained release, TEM

\section{INTRODUCTION}

Prostaglandin E2 (PGE2) treatment assumes an imperative part in the perplexing arrangement of biochemical and basic changes required in cervical maturing. Initiating of PGE2 in vaginal suppository form (pessary) assumes a part in the process of giving birth enlistment. Many reviews infer that sorted out release PGE2 vaginal pessary prompts obvious cervical aging and often starts dynamic work with practically no requirements for oxytocin.

The upsides of PGE2 incorporated patient acknowledge that a lower agent rate than oxytocin, and fewer requirements for oxytocin growth when used with an unfavorable cervix (Bishop $<7$ ). Cost-saving might be acknowledged by a reduction in operative deliveries and/or lengths of stay. PGE2 is a bronchodilator and is not contraindicated in women experiencing the ill effects of asthma. In a forthcoming investigation of 2513 ladies with known asthma and who got PG, none had confirmation of a compounding of their condition [1].

Hydrophilic matrix is the least complex and most financially savvy strategy for manufacturing an extended release (ER) solid oral dosage form. The financially accessible matrix dominant part definitions are as tablets and their assembling technique is like conventional tablet formulations: granulation, mixing, pressure and coating. In the simplest form, a regular ER formulation comprised of a medication at least one water-swellable hydrophilic polymer, excipients, for example, binders, lubricants, other functional ingredients, for example, buffering agents, stabilizers, solubilizers and surfactants, may likewise be included to enhance the release as well as stability execution of formulation system. Different water dissolvable or water swellable polymers with high molecular weight have been used in hydrophilic matrices, for example, hydroxypropyl methylcellulose (HPMC), Polyethylene glycol (PEG) and span 60 [2-5].

*Corresponding author. E-mail: habibmahtook@yahoo.com

This work is licensed under the Creative Commons Attribution 4.0 International License 
HPMC is a mixture of alkyl-hydroxyalkyl cellulose ether containing methoxyl and hydroxyl propyl groups. It is chosen in the prostaglandin E2 passery readiness on the grounds that various key elements, for example, worldwide administrative acknowledgment, excellent stability and no ionic nature, bringing about $\mathrm{pH}$-independent execution, simplicity of manufacturing, adaptability, and appropriateness for different medication and release profiles (on account of various sciences and viscosity grades being accessible), extensivelyexamined and comprehended, and promptly available [6-8]. The formulation advancement work planned to build up a steady formulation of PGE2/sustained release pessaries. Improving the work concentration on the managed release drug delivery production system which preferred dosage form of the medication having a short half-life (under $5 \mathrm{~min}$.), to keep up the medication plasma level in the therapeutic index for a delayed period of time.

\section{EXPERIMENTAL}

\section{Materials}

Prostaglandin E2 93\%, HPMC (400 cP), Witepsol H15, polyethylene glycol (PEG) of subatomic weights of 6000,4000 and 400, sorbitanmonostearate (span 60), and cholesterol $(\mathrm{CH}$; $>99 \%$ ) were acquired from Sigma Chemical Co., St. Louis, MO, USA. Spectrapore ${ }^{\circledR}$ nitrocellulose films (MWCO 2000-15,000) were acquired from Spectrapore Inc., NY, USA. The various fixings used all through the study were of logical and were used as established.

Preparation of $P G E 2$ pessaries

Conventional pessaries. Pessaries containing $10 \mathrm{mg}$ of PGE2 were set-up by the fusion technique utilizing a metal shape with six depressions. WH15 and PEG with various preparations mentioned in (Table 1) were used as pessary bases. Drug displacement estimations of the bases used were initially predetermined and the medication's amount required was ascertained

HPMC-containing pessaries. HPMC in various concentrations $5-30 \% \mathrm{wt} / \mathrm{wt}$ were consolidated into suppositories of WH15 or PEG bases by fusion method.

Span 60/cholesterol containing pessaries. Span 60 with or without cholesterol was liquefied with WH15 or PEG then the calculated medication sum was gradually included and mixed persistently. The amphiphilic mixture/base proportion kept at 1:1. The homogeneous melt was then poured into the mold and left to solidify at room temperature. The presence of amphiphile like Sp 60 in a fatty base could bring about a form we called emulsifying sort suppositories [9]. Then again, PEG is a water solvent base and the presence of amphiphile could bring about niosomal vesicles upon hydration, thus, this form could be named proniosomal suppositories.

\section{Testing of the prepared pessaries}

Uniformity of the weight (B.P. 1993) [10]. 20 pessaries were measured separately, then together figured out and the normal weight as per the following equation:

Average weight $=$ Total weight of the 20 pessaries $/ 20$

There must be not more than two pessaries differ from the average weight by more than $5 \%$ and no suppository varies from the normal weight by more than $10 \%$. 
Melting point. The time lapsed for the whole suppository to dissolve or scatter when soaked in a water bath kept up at $37+1{ }^{\circ} \mathrm{C}[11]$.

Hardness of the pessaries (breaking test). The Erweka technique was used to gauge the weight (in kilograms) of a suppository to endure without breaking. For acceptable outcomes, hardness ought to lie between 1.8 and $2 \mathrm{~kg}$ [12].

Determination of $\mathrm{pH}$ of pessaries. The suppository was processed in warm water, then separated and the $\mathrm{pH}$ of the filtrate was measured by the suitable $\mathrm{pH}$ meter.

Differential scanning calorimetry (DSC) studies. The DSC studies were completed utilizing the differential scanning calorimeter (Perkin-Elmer Pyris 1 DSC).

Electron microscopy. The niosomal vesicle development was demonstrated by electron microscopy (Joel Jim 1010, Japan). The hydrated suppository suspension was used for testing. A niosomal vesicles drop was gone up against a carbon coated matrix. The vesicles were stained utilizing negative stain (uranyl acetate $2 \% \mathrm{w} / \mathrm{v}$ ). The matrices were then envisioned by a transmission electron microscopy (TEM).

Determination of prostaglandin E2 entrapment efficiency

The PGE2 percentage entangled in niosomes formed after the entire hydration of proniosomal pessaries was measured by dialysis technique. The proniosomal suppository was scattered in 5 $\mathrm{mL}$ phosphate buffer of $\mathrm{pH} 4.5$ at $60{ }^{\circ} \mathrm{C}$ and the resultant niosomal scattering is acclimated to 10 $\mathrm{mL}$ of phosphate buffer of $\mathrm{pH} 4.5$ then cooled to room temperature. $1 \mathrm{~mL}$ of the niosomal scattering was put into a dialysis cellophane pack mounted in $100 \mathrm{~mL}$ of phosphate buffer of $\mathrm{pH}$ 4.5 and dialyzing thoroughly for 24 hours at ambient room temperature and magnetic mixing. The entrapment efficiency percent (EE \%) of a PGE2 was measured according to the following Equation:

$$
E E \%=\frac{\text { amountofdrugentraped }}{\text { totalamountofdrug }} \times 100 \%
$$

In vitro release study

In vitro release tests were completed according to a modified USP XXII basket method. Every pessary was set in a glass tube opened on one side and the opposite side was fixed by a cellophane film by means of an elastic band. Around $2 \mathrm{~mL}$ of phosphate buffer $\mathrm{pH} 4.5$ was added to donor side and the tube was suspended in place of the basket in the dissolution apparatus. The glass tubes are then brought down into a flask containing $100 \mathrm{~mL}$ of phosphate buffer ( $\mathrm{pH} 4.5$ ) kept up at $37+0.5^{\circ} \mathrm{C}$ and pivoted at $50 \mathrm{rpm}$. Around $2 \mathrm{~mL}$ samples were pulled back at fitting time intervals and supplanted instantly by $2 \mathrm{~mL}$ of phosphate buffer $\mathrm{pH} 4.5$ to keep up sink conditions. The measure of PGE2 released by the time was examined spectrophotometrically at $272 \mathrm{~nm}$. The experiment was repeated three times and the outcomes were calculated as the mean $\pm \mathrm{SD}$.

\section{RESULTS AND DISCUSSION}

\section{Physicochemical properties}

The delivered pessaries were thin, flat, polymeric pessary (vaginal insert) which is rectangular fit as a shape with adjusted corners. The consequences of the crack point (hardness test), the softening time, the weight consistency, the substance consistency and the medication appropriation inside every pessary was discovered to be satisfactory and presented in Table 2 . 
Table 1. Composition of suppositories of different formulations.

\begin{tabular}{|l|c|c|c|c|c|c|c|c|}
\hline Formula & WH15 & $\begin{array}{c}\text { PEG } \\
4000\end{array}$ & $\begin{array}{c}\text { PEG } \\
6000\end{array}$ & $\begin{array}{c}\text { PEG } \\
400\end{array}$ & HPMC & Sp 60 & CH & Water \\
\hline WH15 & 100 & - & - & - & - & - & - & - \\
\hline WH15/ 5\%HPMC & 95 & - & - & - & 5 & - & - & - \\
\hline WH15/10\%HPMC & 90 & - & - & - & 10 & - & - & - \\
\hline WH15/20\%HPMC & 80 & - & - & - & 20 & - & - & - \\
\hline WH15/30\%HPMC & 70 & - & - & - & 30 & - & - & - \\
\hline W H15/ SPAN: CH (1:0 ) & 50 & - & - & - & - & 50 & - & - \\
\hline W H15/ SPAN: CH(9:1) & 50 & - & - & - & - & 45 & 5 & - \\
\hline WH15/SPAN: CH (7:3) & 50 & - & - & - & - & 35 & 15 & - \\
\hline W H15/SPAN: CH (1:1) & 50 & - & - & - & - & 25 & 25 & - \\
\hline PEG I & - & 33 & 47 & - & - & - & - & 20 \\
\hline PEG II & - & 60 & - & 40 & - & - & - & - \\
\hline PEG III & - & - & 40 & 60 & - & - & - & - \\
\hline PEG III/5\% HPMC & - & - & 38 & 57 & 5 & - & - & - \\
\hline PEG III/10\% HPMC & - & - & 36 & 54 & 10 & - & - & - \\
\hline PEG III/20\% HPMC & - & - & 32 & 48 & 20 & - & - & - \\
\hline PEG III/ SPAN: CH (1:0) & - & - & 20 & 30 & - & 50 & - & - \\
\hline PEG III/SPAN:CH (9:1) & - & - & 20 & 30 & - & 45 & 5 & - \\
\hline PEG III/ SPAN:CH (7:3) & & & 20 & 30 & - & 35 & 15 & - \\
\hline PEG III/ SPAN:CH(1:1) & & & 20 & 30 & - & 25 & 25 & - \\
\hline
\end{tabular}

Table 2. Physicochemical parameters of the prepared pessaries. Data represented as the mean $\pm \operatorname{SD}(n=3)$.

\begin{tabular}{|l|c|c|c|c|}
\hline Formula & Hardness $(\mathrm{kg})$ & Average wt $(\mathrm{g})$ & Softening time $(\mathrm{min})$ & $\mathrm{pH}$ \\
\hline WH15 & 4.5 & $1.2 \pm 0.3$ & 5 & 10.7 \\
\hline WH15/5\%HPMC & 4 & $1.3 \pm 0.4$ & 20 & 11.7 \\
\hline WH15/10\%HPMC & 4 & $1 \pm 0.2$ & 25 & 11.7 \\
\hline WH15/20\%HPMC & 4 & $0.99 \pm 0.1$ & 35 & 11.7 \\
\hline WH15/30\%HPMC & 4 & $1.2 \pm 0.1$ & 25 & 11.7 \\
\hline WH15/1 Sp: 0 CH & 4 & $1.2 \pm 0.0$ & 25 & 10 \\
\hline WH15/9 Sp: 1 CH & 4 & $0.9 \pm 0.3$ & 25 & 10.8 \\
\hline WH15/7 Sp: 3 CH & 3.5 & $0.99 \pm 0.1$ & 20 & 10.3 \\
\hline WH15/1 Sp:1 CH & 2.5 & $1.02 \pm 0.3$ & 20 & 10.8 \\
\hline PEG I & 2 & $0.9 \pm 0.3$ & $>30$ & 10 \\
\hline PEG II & 2.4 & $1.1 \pm 0.3$ & $>30$ & 10 \\
\hline PEG III & 2.5 & $1.1 \pm 0.3$ & $>30$ & 10.3 \\
\hline PEG III/5\% HPMC & 4 & $0.9 \pm 0.2$ & $>30$ & 10 \\
\hline PEG III/10\% HPMC & 4 & $1.12 \pm 0.1$ & $>30$ & 10 \\
\hline PEG III/20\% HPMC & 4 & $1.05 \pm 0.2$ & $>30$ & 10 \\
\hline PEG III/ 1 Sp: 0 CH & 4 & $1.2 \pm 0.2$ & $>30$ & 11.2 \\
\hline PEG III/ 9 Sp: 1 CH & 0.9 & $1.1 \pm 0.1$ & $>30$ & 10.8 \\
\hline PEG III/ 7 Sp:3 CH & 0.6 & $1.2 \pm 0.1$ & $>30$ & 10 \\
\hline PEG III/ 1 Sp:1 CH & 0.6 & $1.02 \pm 0.2$ & $>30$ & 10 \\
\hline
\end{tabular}

The fracture point of the prepared pessaries was between 0.6 to $4.5 \mathrm{~kg}$ which demonstrated adequate mechanical quality to withstand abrasive forces amid capacity or transportation processes. It was observed to be as low as $5 \mathrm{~min}$ in the event of WH15 base and more prominent than $30 \mathrm{~min}$ for PEG base. HPMC (10\% to $30 \% \mathrm{w} / \mathrm{w})$ has expanded the softening time of WH15 to $25 \mathrm{~min}$ where this time was reduced to $20 \mathrm{~min}$ when HPMC was $5 \% \mathrm{w} / \mathrm{w}$. Additionally, 
WH15/Sp60/CH bases demonstrated softening circumstances lower than $30 \mathrm{~min}$. All PEG suppository bases with HPMC of any concentration or with $\mathrm{Sp} 60 / \mathrm{CH}$ demonstrated high softening circumstances, which were more than $30 \mathrm{~min}$. The softening time is critical for fatty pessaries as it could control the rate of medication release, where as for pessaries of PEG base, as of now solubilized by water and medication release will be sorted out by different added substances, for example, HPMC or Sp60/CH. The entrapment efficiency percentage and irritation scores for the applied pessaries are given in Table 3 and 4, respectively.

Table 3. The entrapment efficiency percentage of prostaglandin PG into niosomes prepared from aproniosome suppositories PEG base was $50 \%$ of the total suppository weight: Data represented as the mean $\pm \mathrm{SD}(\mathrm{n}=3)$.

\begin{tabular}{|c|c|c|}
\hline Sp 60/CH & Average unentrapped PG \% & PG Entrapment efficiency \% \\
\hline $1 / 0$ & $3.1 \pm 0.1$ & $96.9 \pm 0.1$ \\
\hline $9 / 1$ & $17.4 \pm 0.2$ & $82.6 \pm 0.2$ \\
\hline $7 / 3$ & $27.9 \pm 1$ & $72.1 \pm 1$ \\
\hline $1 / 1$ & $34 \pm 1.9$ & $66 \pm 1.9$ \\
\hline
\end{tabular}

Table 4. Irritation scores for the applied pessaries.

\begin{tabular}{|c|c|c|c|}
\hline Formulae & Irritation score & Formulae & Irritation score \\
\hline WH15 & 0 & PEG III & + ve \\
\hline WH15/20\% HPMC & 0 & PEG III/30\% HPMC & $-v e$ \\
\hline WH15/ Sp 60 & 0 & PEG III/ Sp 60:CH $(1: 1)$ & 0 \\
\hline
\end{tabular}
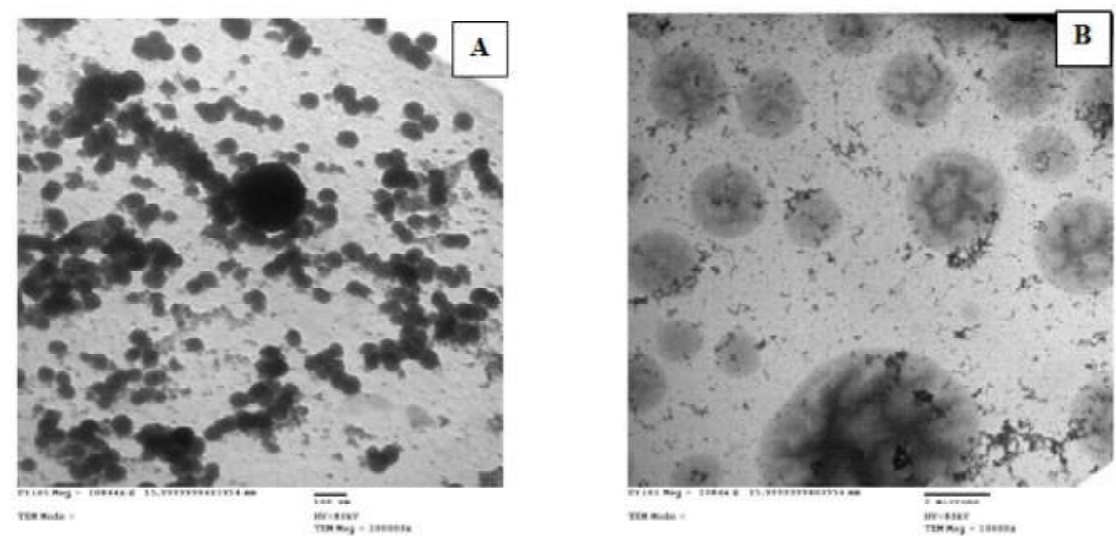

Figure 1. TEM photographs of niosomal vesicles (A) formed from hydrated proniosomal PEG/Sp 60 suppositories and congealed emulsion droplets (B) formed from emulsifying WH15/Sp 60 suppositories with the buffer.

\section{Microscopic examination}

Transmission electron microscopy of the hydrated suppositories at $37{ }^{\circ} \mathrm{C}$ has demonstrated the niosomal vesicles development in the event of PEG/span suppositories or greater emulsion droplets formed when WH15/Sp 60 softened in phosphate buffer. Strikingly, the formed niosomal vesicles were of the unilamellar sort of sizes underneath $100 \mathrm{~nm}$. 
This could be as an after effect of the span surfactant scattering in a high measure of PEG matrix which broke up in the support. This could allow the surfactant particles to total in little numbers and smaller vesicles were created. Then again, WH15/Sp 60 melt cannot form somal vesicles as they are oily in nature and rather emulsion droplets could form which coagulated after cooling forming solid circles as shown in Figure 1. These emulsion droplets could not form with WH15 in the Sp 60 nonappearance.

\section{DSC studies}

The thermogram acquired from these reviews indicates unadulterated PGE2 DSC curves, its polymer physical mixture, and diverse co-excipients. A sharp endothermic top at 67.08 was acquired for unadulterated PGE2 relating to its melting point. The endothermic peaks of the formulation WH15/30\%HPMC and PEGIII/SP60 appeared in 65.03 and 63.86 individually, because of different physical mixture concentrations. Therefore, these minor changes in the melting endotherm in the medication would be attributed to the mixing of the medication and excipients, which reduce every components' purity in the mixture. As PGE2 melting and the details, WH15/30\%HPMC and PEGIII/SP60 are nearer and did not demonstrate real change, representative any possible contact shown in Figure 2.

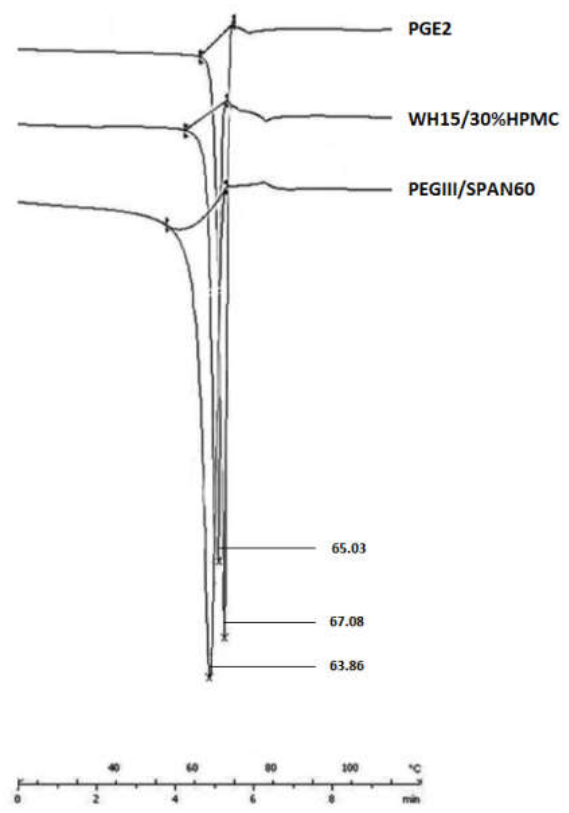

Figure 2. DSC thermogram of pure PGE2, WH15/30\%HPMC and PEGIII/Sp60.

\section{In vitro discharge properties}

The release of PGE2 from various suppository bases is shown in Figure 2. Clearly, the medication is all around released from both hydrophilic and hydrophobic bases. The percentage medication released after $4 \mathrm{~h}$ was $104 \%, 91.3 \%, 88.8 \%$, and $85.1 \%$ from WH15, PEGIII, PEGII, and PEGI, individually. The outcome may be because of the way that PG E2 has a low partition coefficient $(\log \mathrm{P}$ octanol/water $=0.23)$. Thus, it is a profoundly hydrophilic medication [13]. Likewise, it is clear from the outcomes that the distinctions in medication release were not high 
in utilizing a PEG base of different synthesis. There are just $6 \%$ distinction between the most astounding releasing base (PEG III) and the least one (PEG I). In like manner, the PEG III base was chosen to prepare SR suppositories. The outcomes uncovered that both hydrophilic PEG base and lipophilic witepsol bases were appropriate for SR suppository plans for PGE2. With a specific end goal to control the PGE2 release from a suppository base, HPMC and Sp 60/CH were used and assessed.

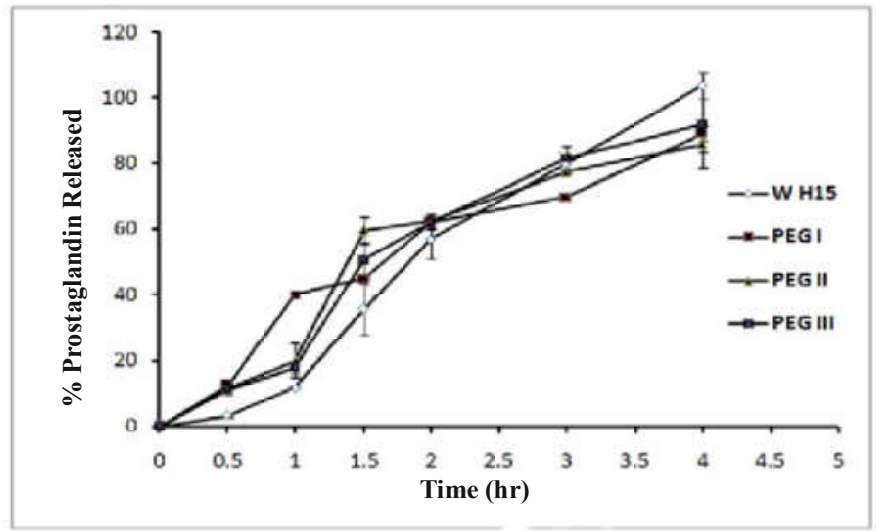

Figure 3. Release prostaglandin from hydrophobic base WH15 versus hydrophilic PEG bases.

Data represented as the mean $\pm \operatorname{SD}(n=3)$.

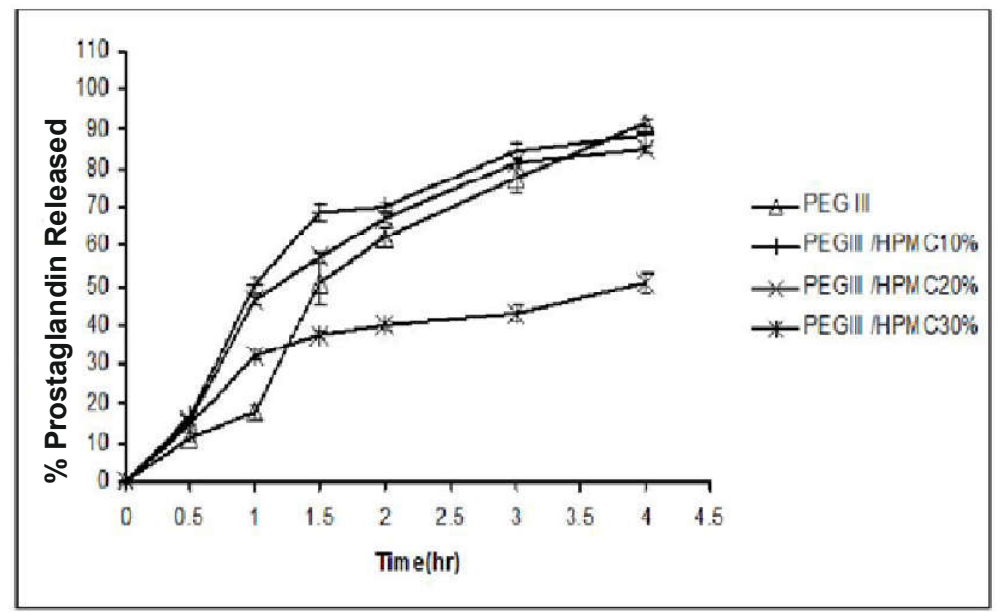

Figure 4. Release prostaglandin from PEG III/ HPMC pessaries. Data represented as the mean $\pm \mathrm{SD}(\mathrm{n}=3)$.

Results are represented in Figures 3 to 6 . The addition of HPMC to a suppository base at $10 \%$ and $20 \%$ concentrations in the PEG III base demonstrated comparable examples to that acquired from the pure PEG III formular. Then again, at 30\% HPMC concentration, the PG E2 release has decreased, especially to around $50 \%$ after 4 hours (Figure 3). The previous results could be because of the expanded hydrophilicity of the PEG base upon HPMC addition at lower 
concentrations, where at high HPMC concentrations, the matrix viscosity may notably increase forming matrix structure and thwart the medication dispersion. The expansion of HPMC to WH15 base demonstrated a more exact example where the PG E2 release was linearly decreased as the HPMC concentration increased. The PG E2 release was decreased from $94 \%$ to $22 \%$ as HPMC concentration expanded from $5 \%$ to $30 \%$ in the WH15 base, separately (Figure 4). As previously discussed [14], the decreased release rates due to a viscosity modifier were ascribed to the most gelling conduct shown by the gelling part.

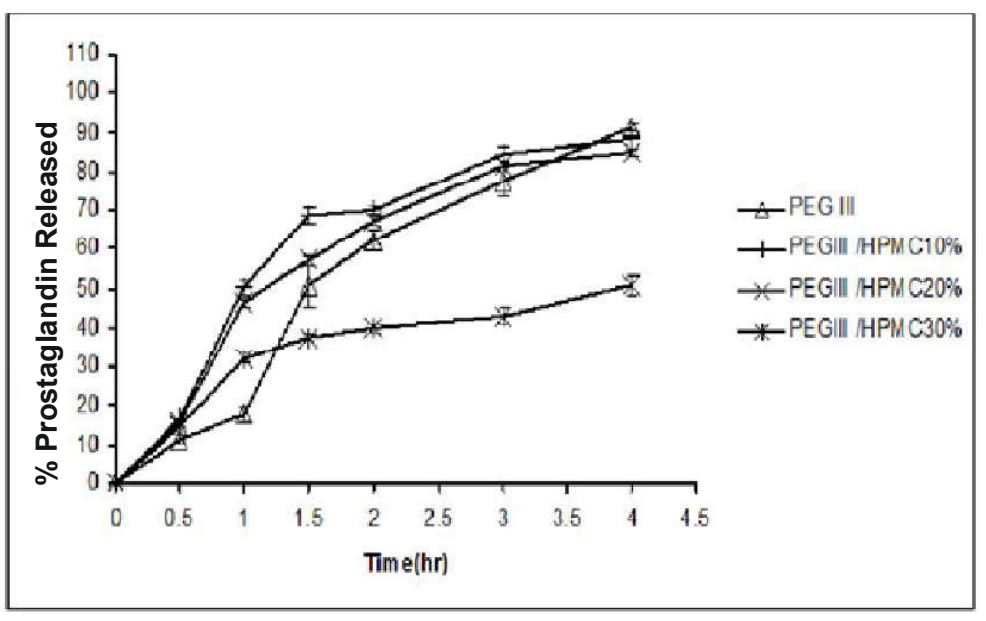

Figure 5. Release of prostaglandin from WH15/HPMC pessaries. Data represented as the mean \pm $\mathrm{SD}(\mathrm{n}=3)$.

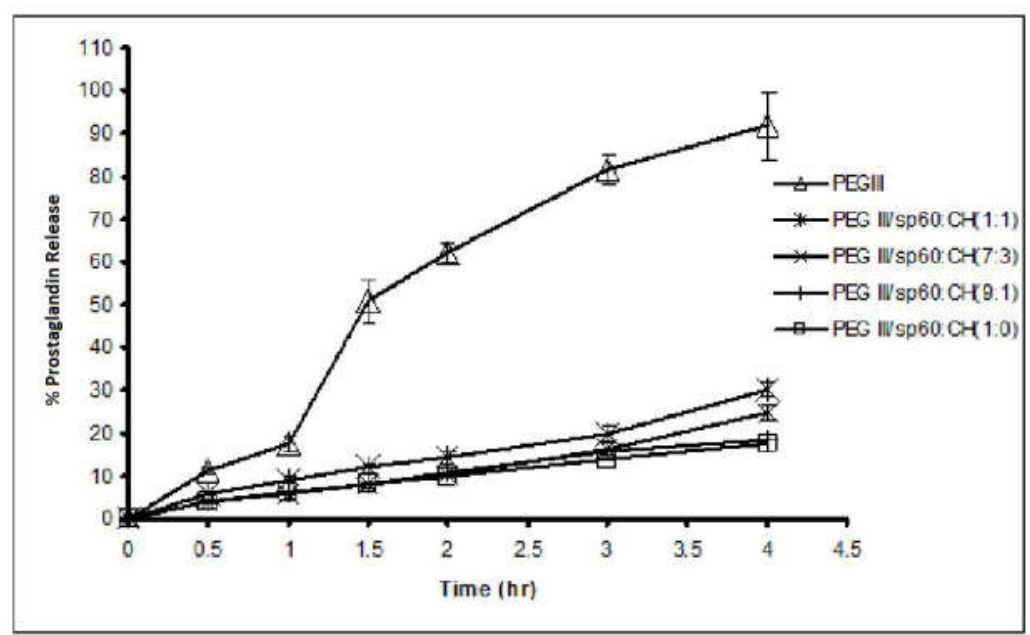

Figure 6. Release of prostaglandin from PEG III/ Sp 60: CH suppositories. Data represented as the mean $\pm \operatorname{SD}(\mathrm{n}=3)$. 


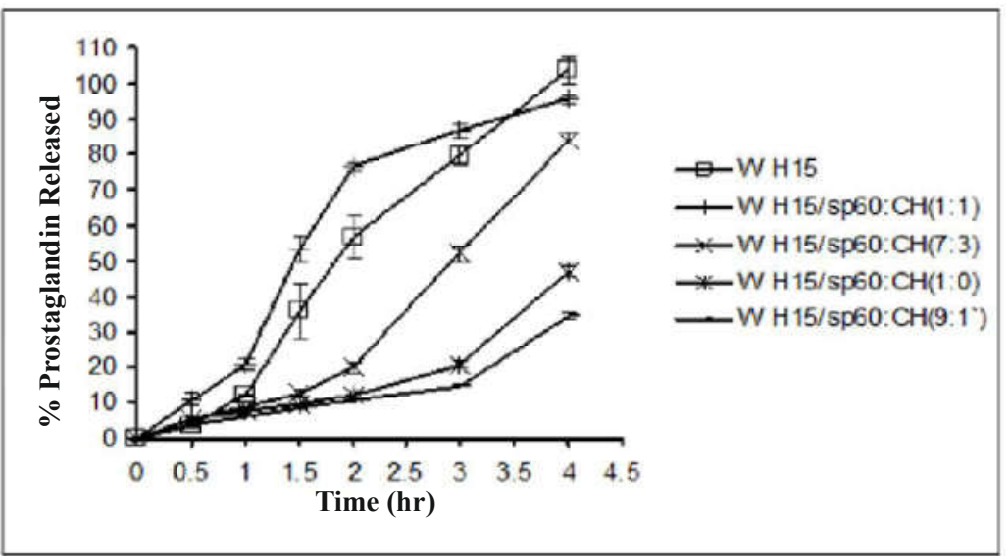

Figure 7. Release of prostaglandin from WH15/Sp 60: $\mathrm{CH}$ pessaries. Data represented as the mean $\pm \mathrm{SD}(\mathrm{n}=3)$.

The HPMC mucoadhesion properties additionally gave the formulation more concentrated guarantee as SR rectal formulation. Sp 60 can self-assemble in water forming bilayer vesicular structures called niosomes [15]. As a medication conveyance matrix, niosomes can hinder medication release by entrapment either in the bilayer structure or inside the fluid compartment. The integration of Sp 60 into the PEG III base brought about great exquisite suppositories with adequate physicochemical properties recorded in Table 2 . The created suppositories could be a proniosome sort where niosomal vesicles were clearly shot after hydration and represented in Figure 1. The entire scattering of the suppository followed by the hot buffer addition brought about niosomes scatterings and the PG E2 entrapment efficiency into niosomes was recorded in Table 3.

From Table 3 it is obvious that the PG E2 entrapment efficiency decreased from $96.8 \%$ to $66 \%$ as the cholesterol rate (figured as a percent of aggregate span molar concentration) increased from $0 \%$ to $50 \%$. The high encapsulation of PGE2 might be ascribed to the medication solubility in the lipid mixture before reconstitution in the buffer which inserts the medication in the lipid bilayer. As PG E2 packed into the bilayer, it could contend with cholesterol for positions in the bilayer structure and this prompted to the decline in medication entrapment efficiencies as cholesterol rates increased [16]. The release of Atenolol from proniosomal suppositories could be clarified on the premise of the medication entrapment efficiency. Figure 5 shows a more prominent decrease in the PG E2 release rate where Sp 60 incorporated into PEG suppositories without cholesterol (around 17\% after 4 hours). The rate PG E2 release increased to $38 \%$ after $4 \mathrm{~h}$ when cholesterol expanded to $50 \%$ of aggregate lipid concentration Figure 5. It is considerable, that as the un-entrapped medication rate increased the rate of PG E2 release increased. The incorporation of Sp 60 into WH15 fatty base could not form niosomesup on hydration because of the abundance of the oily medium which restrains the self-assembly of the surfactant into bilayer structures (Figure 1).

Emulsions could form and the release of PG E2 also influenced. Figure 7 demonstrates a decreased PG E2 release rate from 103\% to 34\% when Sp 60 is added to the WH15 base and in $\mathrm{CH}$ nonappearance. Once more, the option of cholesterol had increased the medication release rate from $34 \%$ at $0 \% \mathrm{CH}$ to $96 \%$ at $50 \% \mathrm{CH}$, in any case, this may be attributed to the increase in the lipophilicity of the base as $\mathrm{CH}$ is added Figure 7. As specified above, the low partition coefficient of the medication prompted to the fast release from fatty bases. 


\section{CONCLUSION}

An organized release prostaglandin pessary (vaginal suppositories) were effectively formulated either by utilizing HPMC as a mucoadhesive polymer, proniosomal technology in hydrophilic PEG or emulsification of WH15 base with Sp 60. The proniosomal sort suppositories, the WH15/Sp 60 and WH15/HPMC suppositories indicated non irritant, organized release and good cervical ripening impacts of prostaglandin, which is superior to both oral and rectal administration of fast release formulae. Finally, this study recommends the prostaglandin formulation into sorted out release pessary by utilizing HMPC, PEG, WH15/SP 60 and WH15/HPMC as bases is good at initiating labor and cervical ripening.

\section{REFERENCES}

1. Towers, C.V.; Briggs, G.G.; Rojas, J.A. The use of prostaglandin E2 in pregnant patients with asthma. Am. J. ObstetGynecol. 2004, 190, 1777-1780.

2. Alderman, D.A. Formulation design of an HPMC-based sustained release tablet for pyridostigmine bromide as a highly hygroscopic model drug and its' in vivo/in vitro dissolution properties. Int. J. Pharm. Tech. Prod. Mfr. 1984, 5, 1-9.

3. Hogan, J.E. Hydroxypropylmethylcellulose sustained release technology. Drug Dev. Ind. Pharm. 1989, 15, 975-999.

4. Pygall, S.R.; Kujawinski, S.; Timmins, P.; Melia, C.D. Mechanisms of drug release in citrate buffered HPMC matrices. Int. J. Pharm. 2009, 370, 110-120.

5. Siepe, S.; Lueckel, B.; Kramer, A.; Ries, A.; Gurny, R. Strategies for the design of hydrophilic matrix tablets with controlled microenvironmental pH. Int. J. Pharm. 2006, 316, 12-20.

6. Rajabi-Siahboomi, A.R.; Bowtell, R.W.; Mansfield, P.; Davies, M.C.; Melia, C.D. Structure and behavior in hydrophilic matrix sustained release dosage forms: 4. Studies of water mobility and diffusion coefficients in the gel layer of HPMC tablets using NMR imaging. Pharm. Res. 1996, 13, 376-380.

7. The Dow Chemical Company. Methocel Products; 2008.

8. Gao, P.; Skoug, J.W.; Nixon, P.R.; Ju, T.R.; Stemm, N.L.; Sung, K.C. Swelling of HPMC matrix tablets. II. Mechanistic study of the influence of formulation variables on matrix performance and drug release. J. Pharm. Sci. 1996, 85, 732-740.

9. Hardy, I.J.; Cook, W.G.; Melia, C.D. Controlled release in oral drug delivery. Int. J. Pharm. 2006, 311, 26-31.

10. Huang, Y.; Khanvilkar, K.H.; Moore, A.D.; Hilliard-Lott, M. Effects of manufacturing process variables on in vitro dissolution characteristics of extended-release tablets formulated with hydroxypropyl methylcellulose. Drug Dev. Ind. Pharm. 2003, 29, 79-88.

11. British Pharmacopoieal Convention. British Pharmacopoeia, Vol. 2, H.M. Stationery Office: London; 1993.

12. Moorthi, D.D.; Jawahar, N.; Jayprakash, S. Design and evaluation of sustained release suppositories of nimesulide. Ind. J. Pharm. Sci. 2005, 67, 558-561.

13. Dorle, C.D.; Jaiswal, S.B.; Mandavgade, S.; Purohit, R.N.; Sakarkar, D.M. Comparative studies and pharmacokinetic evaluation of rectal suppositories of diltiazem hydrochloride in rabbits. Int. J. Pharm. Excip. 2003, 55-60.

14. Noro, S.; Kamatsu, Y.;Uesugi, T. Influence of surfactants, polymers and concentration of the water phase on in vitro drug release from emulsion type suppositories. Chem. Pharm. Bull. 1982, 30, 2912-2918.

15. Abass, H.; Kamel, R.; Abdelbary, A. Metronidazole bioadhesive suppositories: Formulation, in vitro and in vivo evaluation. Int. J. Pharm. Pharm. Sci. 2012, 4, 344-353.

16. Uchegbu, I.F.; Vyas, S.P. Non-ionic surfactant based vesicles (niosomes) in drug delivery. Int. J. Pharm. 1998, 172, 33-70. 